\section{Ann Adùress}

\author{
ON
}

\section{THE INDICATIONS FOR, AND LIMITATIONS OF, SURGERY IN CHRONIC ABDOMINAL \\ DISEASE. *}

JAMES SHERREN, C.B.E., F.R.C.S., SURGEON TO THE LONDON HOSPITAL, ETC.

IN speaking of the indications for, and limitations of, surgery in chronic abdominal disease I am using the word "surgery" to mean operative surgery-in itself a limitation, as this form of treatment involves the removal of organs or parts of organs, their drainage, short-circuiting, or fixation. We appear to have reached a stage at which operative technique lias progressed moro than diaguosis, and treatment is often in advance of, and may be more severe and dangerous than, the "disease" for which it is recommended.

Surgery, of course, is not so limited and cannot be dirorced from medicine. The surgeon needs to be a physician, and it riglitly falls to his lot to suggest and supervise courses of treatment other than operative. At the present time, howerer, most patients are sent to him when rest, diet, and drugs have failed to relieve, to see if by his art he can remove tlie cause of the symptoms or in some way alter the relation. ships of organs that it ceases to act.

Our aim should be to discover the cause of the disease, and so prevent the development of lesions necessitating for their treatment mutilating operations, such as remoral of the gall bladder, sp'een, portions of the stomach or intestine. We know that long life in comfort and perfect health is possible after all these procedures, but he would be a bold man or surgeon who would assert that the patient who has lost portions of his internal anatomy or whose internal pliysiology vas altered by short circuits is in no way worse off thau one not so treated, although we know that he is infinitely better off ivhen disease has been cured or relieved by these means.

It may be with us surgeons as with the sailor's of the last ccntury. When the art of building sailing ships and sailing them reached its zenith, so the need for thesc arts declined, and is now almost lost. It is possible that operative skill will become similarly unnccessary, except in dealing with the result of accidents.

We should not be obsessed with the idea that there must be an operative cure for all abdominal ills, and allow the fact that many abdominal operations can be done at small risk and with little discomfort to lead us to perform or advise operations on speculative grounds.

There is no doubt of the necessity of operation at the present time in dealing with many forms of chronic abciominal discase. In malignant disease its localization is an indica. tion, its secondary deposits a limitation. Although we are still ignoraut of its cause, I have no doubt that in the abdomen its onset may be prevented in many cases. 'There is a preventivo surgery as well as a preventive medicine. 'Timely appendicectomy will, I am convinced, anticipate the develop. ment of many ulcers of stomach and duodenum and disease of the gall bladder; but the appendix must be a diseased one-wholesale removal of this organ in cases of visceroptosis, sacro-iliac strain, etc., only brings surgery into disrepute. The early and efficient treatment of acute gastric ulcer may hinder its. change into the cluronic type, and early operation on the latter will certainly prevent later malignancy. Similarly, removal of the diseased gall bladder will do away not only with the risk of recurrence of gall stones, when undertalsen in cases with this complication, but the possibility of the later development of carcinoma-a risk by no means negligible following cholecystostomy. Drainage of the gall bladder is not curative; all sufficiently diseased to nced operation need removal, and there is no more satisfactory operation in surgery that can be undertaken at so slight a risk.

It is essential that diagnosis nrecede treatment, although that diagnosis may not bo made until the abdomen has been opened, but no lesion diagnosed then on symptoms only should ever be treated by surgical procedure; no organ should ever be removed or any mechanical alteration made except for disease that can be definitely recognized-never for symptoms thought to be attributable to a certain organ.

Although diagnosis may be impossible until the abdomen

* Given at the Annual Meeting of the Suffolk Branch of the British Medical Association on June 30 th has been opened, every endeavour must bo made, by every method at our disposal, to come to a pre-operative decision as to the cause of the symptoms. We must at any rate be ablo to say, Surgery will benefit this patient; there is a lesion here that needs operative treatment. It should not be a case of look and see if anything can be done. We should blush to find on opening the abdomen that no demonstrable lesion is present.

In abdominal surgery attention must not be directed only to the organ thought to be at fault; the viscera have interrelationships. When operating for chronic disease the who!e abdomen must be explored, and the exploration must bo thorough. I have frequently given examples of this and have often had to operato on patients with disease of the gall bladder, stomach, or duodenum in whom $I$ have been informed that this was absent because an incision had been made, the appendix removed, and these organs investigated by touch. It cannot be said too ofteu that disease of the gall bladder -including one of its results, stones-cannot al ways bo diagnosed by touch, and it is often necessary to open it and inspect, even to discover these. Only last week I operated on two patients ; in both the gall bladder to the onlooker looked healthy. In one there was thick bile, cholesterin deposits in its mucous membrane, and polypoid cholecystitis; in the other, stones that could not be felt externally. If I suspect gall-bladder disease I always oren it and investigate.

Again, how many ulcers of the duodenum or stomach is it possible to feel with the tips of the fingers from below, and how many are missed through making a small incision and exploring digitally? Surgeons should not limit themselves in this way. We used to pride ourselves on being able to remove an appendix through an inch incision; it can be done, but is not surgery.

In treating chronic gastric and duodenal u!cer we should remember that we are dealing with the end-result of disease of which we can at present only discern dimly the cause. The future treatment I believe will not be operative; its indication now is failure to cure or alleviate by medical means. This includes treatment of all foci of infection-rest in bed, diet, etc. ; when all these fail surgery is indicated. We must realize that this necessarily involves interference with the physiology of digestion, whether the operation is a short circuit or remoral of a portion of the stomach. Operative success in treatment is impossible without altering normal relationships, and the human organism has to adapt itself to it, and does so very well.

Each disease has its own dangers, and operation is indicated on failure of medical treatment because of the grave risk in chronic gastric ulcer of the development of carcinoma, of haemorrhage and less, of perforation. The risk of the derelopment of carcinoma is a very real one. It is no uncommon thing to find on operating on patients with long standing histories pointing to gastric ulcer that carcinoma has supervened and is now beyond the skill of the surgeon. I have found, in a fairly extensive operative experience, that in at least 45 por cent. the carcinoma has followed on chronic gastric ulcer. Others put the figure much higher. Haemorrhage from chronic ulcer is a complication second in severity only to perforation, and one that should be prevented by timely surgical intervention. When it has occurred, operation should be carried out so soon as the condition of tho patient will admit. I like to do it within twenty-four hours of the first attack. Many of these ressmble secondary haemorrhage, with which you were unfortunately so familiar during the late war; after a warning there is a latent period followed by haemorrhage which not infre. quently continues to a fatal issue. I am speaking now of haemorrhago in patients suffering from chronic ulcer-that is, in those with a long-standing history of attaclss of indigestion separated by periods of liealth. It does not apply to haematemesis arising in some cases from acute ulcers, that can be conveniently grouped under the heading of gastro. staxis. These do not need operative treatment. We must not, however, let ou'selves be hypnotized by such a word, but always be on the lookout to recognize the cause in each case, and remember that an acute haematemesis is sometimes the first symptom of carcinoma of the stomach and the first symptom attracting attention in splenic disease amenable to surgical treatment.

Operation is advisable in cluronic duodenal ulcer, because of the great risk of perforation and haemorrhage. All who have had much experience in the treatment of this grave disease will recall instances in which perforation occurred during a period of latency, betwcen the attacks, when the 
patient considered himself well. No one with this disesse, whatever precautions he may take, can consider himself safe from this complication. In many patients the attacks may not interfere much with their life, but it is the risk that makes me advise operation in every case that has resisted efficient medical treatment.

After operations for chronic gastric or duodenal ulcer, notwithstanding altered relationships, the patient is relieved in the majority of cases, and in 90 per cent. is cured, whether the treatment applicable has been posterior gastro-jejunostomy or partial gastrectomy. Failures there must be in every thing in this life, in surgery as in all other mundane affairs these are our limitations, and are mostly due to the impossibility of discovering a still active causative agent, and the recurrence of ulceration in the region of the new opening after operation for chronic duodenal ulcer, or, in still rarer instances, to mechanical changes which may arise in the region of the anastomosis due to the formation of adhesions and resulting in vomiting.

The indications for removal of the appendix are obvious in chronic disease of the type associated with attacks of genera abdominal pain with tenderness in the right iliac fossa. It is in these cuses that it is justifiable to operate through a small muscle-splitting incision.

It is still necessary to remonstrate against removal of the appendix for pain confined to the right iliac fossa or right side of the abdomen; this is still being diagnosed as due to the appendix, and this organ is being removed without success in its treatment. Hardly a week passes in which I do not see patients in whom this operation has been done unnecessarily and unsuccessfully. Appendicectomy is now so safe that there is very great danger of it being carried out indiscriminately for discomfort rather than for disease and after insufticient investigation. A pain in the right side and tenderness on palpation are not sufficient justification for operation.

It is at present one of our limitations that we have not discovered a satisfactory cause for many of the pains in the right iliac fossa, met with particularly in women. Kidney diseases, ovarian tumours and tubal disease, atony and prolapse of the caecum, intestinal stasis may all be considered responsible, and if due to the first three groups can be cured by surgical means; it is doubtful, however, if this is possible with the remainder; in the majority of these. We cannot discover any really satisfactory cause. All, however, need the closest investigation, and care must bo taken not to overlook pain due to disease of the kidney and to chronic obstruction, particularly in the pelvic colon, in which the pain may be confined to the right iliac fossa.

Removal of the appendix cannot be expected to cure disease of the gall bladder, stomach, and duodenum; yet how often do we see patients with obscure abdominal symptoms in whom the appendix has been remored through an incision which makes thorough abdominal exploration impossible. Our tactile sense will not diagnose early, and sometimes even late, con. ditions here. I have long taught, and will once again repeat it: You may diagnose appendix dyspepsia, you should never act on it-that is, you should never remove the appendix alone in these cases and wait for events, but examine every other abdominal vigcus. These are the cases of chronic appendicitis in which it is not justifiable to operate through the muscle-splitting incision in the right iliac fossa. For example, last weel I operated upon a male patient of 25 with a history of attacks of indigestion for four years, whom I had been asked to see as a case of appendix dyspepsia. The report of the barium meal was a normal stomach; his test meal gave normal gastric acidity. I removed a thickened appondix becoming obliterated at its distal end and then prolonged the incision upwards. I found a chronic ulcer on the lesser curvature about an inch from the pylorus. This is the most recent example, but I have had a similar experience on many occasions, on more have had to reoperate after others, and have found not only chronic ulcers and gall-bladder discase, but even carcinoma of the stomach, after the appendix had been removed on the diagnosis of appendix dyspepsia.

It is one of the limitations of abdominal surgery at the present time that it is impossible to exclude disease without examining by sight and touch the suspected organs. When abdominal symptoms are present they raay be definite enough for us to say they are due to disease of the gall bladder, the duodenum, the stomach, or the appendix, but we cannot say that there is no disease in either of the other organs without looking. In the type of case, by no means uncommon, in which, in spite of every method of investigation, we are uncertain of our diagnosis, all have equally to be examined. Our limitations will be fewer if we remember that the days of small incisions are over. If a paramedian incision is used, pulling the rectus out its length does not matter. I would remind you that in chronic-abdominal disease the organs with which we liave to deal are situated principally to the right of the mid-line, yet I still see patients wiro have had a " gastro-jejunostomy" performed or even an exploration carried out through a left. sided incision.

When dealing with the colon one enters at once into a region where there has been and still is fierce con. troversy. We are, however, on absolutely solid ground when we give as an indication for surgical treatment the removal of portions of the colon that are so diseased as to be incapable of performing their function. The operative surgery of malignant disease here, as elsewhere, has its limitations in spread of the disease outside the limits of the organ; the problem of treatment consists in removing sufficient bowel together with its lymphatic area of drainage. In non-malignant conditions every endeavour should be made to conserve the colon, and, if excision is necessary, to remove as little as possible. In dealing with the caecum and ascending colon we must bear in mind that removal of the ileo-caecal valve profoundly alters intestinal digestion. Removal of the colon or its right half must interfere seriously with the functions of the alimentary tract. The colon is necessary in the human economy; it should never be removed unless its retention threatens life.

In chronic ulcerative diseases not cured by medical measures appendicostomy in my hands has given brilliant successes, but there are failures. In these I have brought up a coil of the ileum about a foot from the ileo-caecal valve and drained it so as to give the colon absolute rest, and not restored the continuity of the alimentary canal until examination of washings has proved the ulceration to be healed. Ileo-sigmoidostomy is useless in the treatment of ulcerative colitis; it is an operation that is rarely, if ever, justifiable; it does away with the action of the ileo caecal valve, and does not prevent overfilling of the colon proximal to the anastomosis. If a short-circuiting operation is necessary in the treatment of irremovable carcinoma of the colon it should be colon to colon.

We come now to a group of cases with which all are familiar, under the term "visceroptosis." Much more often met with in women than in men, it is a continual source of invalidism, if not of real.ill health. It is therefore not surprising that patients seek, and surgeons have endeavoured to. find for them, relief by operative means. Many are the operations that have been devised and carried out: division of adliesions, plications, fixation of stomach and colon, even to colectomies, partial or complete, witl, I believe, no more success than will attend a similar compulsory rest in bed. The more experience I have in abdominal surgery the less success do I expect from operation in these cases and the more loath am $\mathbf{I}$ to undertake it. We are driven to it sometimes by the limitations of our knowledge; it may be quite impossible to exclude other and more serious disease without exploration. Gall-bladder disease is common in this type of case. Clironic gastric ulcer high on the lesser curvature is not infrequent, and carcinoma may develop.

Always remember that it is not a disease that shortens life; carry out nothing that may do so. I believe many of these patients are suffering from disease of their autonomic nervous system - that the visceroptosis, the dropped stomach, atonic and prolapsed colon are not causes. They may all exist without producing symptoms, and those that occur cannot always be explained by their presence. Diagnosis las outruu treatment here. Surgeons were justifiably eager to seize upon these "misplacements," just as in earlier years displacements of the uterus were looked upon as the source of clironic ill health. From the experience we have gained in its surgical treatment $I$ believe that in its management such operations as fixation of colon, partial colectomy, plication of caecum, will disappear.

We must realize our limitations here, while trying in every way to improve our methods of diagnosis. There are still pains and discomforts in the abdomen that cannot be re. moved with the appendix or by fixing viscera or difiding adhesions.

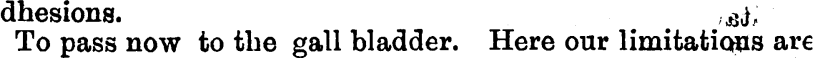
less, as we are able to deal with the disease and not only remove its results. At the present time, only operation car demonstrate the condition of the gall bladder. I would agair lay particular stress on the fact hat until the gall bladdes 
has not only been seen but explored by opening, it is impossible to be certain that disease is absent. In the early stages of gall-bladder disease cure by surgery is sure and safe; in the presence of such complications as stone in the common duct and acute infections the risk becomes greater, and malignancy may be the termination of- chronic simple gall-bladder disease.

When the gall bladder is diseased its removal is indicatedin two stages, if life or the integrity of the common duct is threatened if done in one. Draiuage alone will not certainly cure, nor will it prevent the later onset of carcinoma in gall bladders believed, on external examination, to be healthy at the time of drainage, after removal of calculi. I have known it to arise from one to four years after drainage of what was considered a sufficiently healthy gall bladder to leave after the removal of calculi.

One of the most recent advances in abdominal surgery is that dealing with disease by splenectomy. Although we know that removal of the spleen is followed by restoration to healtl in suitable cases, until we are fully cognizant of its functions we cannot understaind the part it plays in disease and why removal in certain cases is efficacious. That long life was possible after removal of the spleen in man we owe to clinical observation after splenectomy for injury. Interference next followed in such conditions as wandering spleen with torsion of the pedicle or the rare tumours. More recently, diseases of the spleen associated with blood changes have been successfully treated; I allude to such groups as splenic anaemia, Banti's disease, and splenomegalic jaundice. In the former, restoration to health occurs, and so far as we can ascertain no evil effects follow; we can justifiably speak of cure. It may be otherwise when operation is carried out in the later stage of the disease when cirrhosis of the liver has supervened. With this complication, when haemorrhages have taken place before operation, although apparent restoration to health is the rule, yet death from haematemesis at a later date is not unknown, in spite of the fact that splenectomy has been suggested as the treatment for certain cases of haematemesis in the absence of gastric or duodenal ulcer, splenomegaly, or recognizable hepatic cirrhosis. Our limitation here is due to neglect of treatment in the early stages of the disease.

Of the cause of the disease and the splenic enlargement, whether due to thrombosis-and if so, the infecting agent and its organ-we are in ignorance. Why removal of the spleen alters the blood picture is also beyond our ken. We are sadly limited in our dealings with diseases associated with splenic enlargement in not lnowing their cause.

I know of no condition in which recovery is so dramatic as in splenomegalic laemolytic jaundice. In the familial type discoloration may have persisted from the early years of life, and yet within forty-eight hours of removal of the spleen it has disappeared. The fragility of the red cells, part of the disease, may be unaffected by splenectomy. As in other chronic abdominal diseases, general exploration has to be carried out, particular attention being paid to the gall bladder and ducts, as these cases are often complicated by pigment caleuli.

Splenectomy in pernicious anaemia and in leukaemia. is experimental, and until more evidence is forthcoming we should stay our hand.

Surgery has many legitimate triumphs in the abdomen. Let us see to it that it is not brought into disrepute by operations for trivial complaints, and that removal of the appendix or gall bladder, - or fixations of the colon, do not become common procedures in every discomfort in this region.

\section{PAIN IN GENITO-URINARY AFFECTIONS:} ITS VARIATIONS AND THEIR INTERPRETATION.

BY

W. K. IRWIN, M.D., F.R.C.S.EDIN.

HONORARY CASUALTY OUT-PATIENT SURGEON, ST' PAUL'S HOSPITAL FOR GENITO-URINARY DISEABES.

The variations of pain in genito-urinary affections are so numerous and often so misleading that their interpretation may be difficult. It is hoped that the following notes will facilitate the early recognition of the causes at work.

Paif arising in this system frequently shows itself in parts other than the seat of the lesion, and it may be well at the outset to emplrasize the distinction between (1) site pain, which is felt in or over the-lesion, and (2) referred pain; which through abnormal perception by the senses is assigned to the region supplied by an anastomosing nerve trunk or to the distribution of an irritated nerve.

Renal and Ureteric Regions.

Affections of the kidney are comparatively painless if the ureters remain patent. Renal calculi may give rise to three varieties of pain: (1) site pain, (2) referred pain, - (3) renal colic. The site pain, which is more prominent during the diurnal hours of activity, is a dull ache, and is felt in the front of the loin, in the posterior renal angle behind, or in both places. The referred pain may be assigned to the healtlyy kidney (reno-renal reflex), testicle or labium, bladder, urethra, thigh, knee, calf, inside of foot, sole or heel, and mav be of a burning nature. In renal colic the pain, which may be described as "tearing or griping," is characterized by agonizing paroxysms radiating down the line of the ureter and along the urethia, or referred to the external abdominal ring and into the testicle, causing contraction of the cremaster muscle.

Oxaluria may cause unilateral pain similar to that of renal calculus. Such pain as there may be in tuberculous kidney is a dull lumbar ache described as toothache-like, though tuberculous débris in a ureter may cause ureteric co!ic. Movable kidney is characterized by a dragging ache, aggra. vated by movement, but the kinking of a ureter in a Dictl's crisis may produce pain resembling the colic caused by a calculus. In kidney tumour pain, which is not increased by movement, may result from increased intrarenal tension, from ureteral obstruction by blood clot, or from pressure on surrounding stractures. Ureteral calculi usually give rise to symptoms akin to those of renal calculi, the pain being due to the distension and spasmodic contraction of the kidney pelvis and of the ureter above the stone rather than to the irritation produced by the stone itself. Pain of a similar cliaracter may be caused by an aberrant blood vessel, by stricture or kinking of the ureter, or by its becoming blocked by tuberculous débris, blood clot, or a villous growth of the kiducy pelvis.

Vesical and Urethral Regions.

Apart from acute blocking of the ureter genito-urinary pain as a rule has its source in the bladder, prosíate, or prostatic urethra. Pain originating from the upper half or more of this bladder is usually felt above the pubis, but pain from the ureteric orifices and adjacent part of the bladder wall is sometimes referred to the corresponding inguinal ring or along the corresponding side of the penis. Perineal pain suggests disease of the vesical base or neck, of the prostate, prostatic or membranous urethra, or of some structure intimately associated with one of them, constant cluronic pain being in most cases due to prostatitis, or to carcinoma or calculus of the prostate, and pain increased at the end of mictarition to calculus, inflammation or ulceration of the bladder; but in any of these conditions the pain may be referred to the end of the penis. Urethral pain if before micturition is usually caused by obstruction from impacted calculi, etc.; if only during micturition, by inflammation or ulceration of the urethra.

Pain due to vesical-calculus occurs principally at the end of micturition, and may be described as burning and stinging or sharp and cutting. It is usually felt at the neck of the bladder and in the urethra slightly behind the external meatus, but may be felt in the perineum and at the root of the penis. In children it is often very severe, the patient screaming and pulling at his foreslkin, especially at the end of micturition; but in old patients if the stone lies. in a post-prostatic pauch, so as not to come in contact with the vesical neck, little if any pain may be felt. The pain may graduaHy decreaso owing to the calculus becoming larger and smoother by the deposit of phosphates around it. 'The eff sct of rest upon pain due to vesical calculus is so great as to bo an important diagnostic sign.

Such pain as is caused by calculi of the prostate is usually felt only during and after micturition, but there may be a constant ache increased by defaecation and unconnected witl micturition, while in calculi of a seminal vesicle painful defaecation and seminal ejaculation may occur. Acute cystitis produces scalding pain.along the urethra during micturitiou, while at the end of the act, or. if the patient tries to hold his water, there may be pain in the region of the vesical neck. In tuberculous cases severe pain as a rule indicates ulceration of the trigone or the onset of mixed infection. The features of pain in acute urethritis and prostatitis are too familiar to need any description: Acute spermatocystitis is associated 